\title{
Hammett spectral correlations in some aryl 1,3-oxazine-4-thione derivatives
}

\author{
G. Thirunarayanan ${ }^{1, *}$, K. G. Sekar ${ }^{2}$ \\ ${ }^{1}$ Department of Chemistry, Annamalai University, Annamalainagar - 608 002, India \\ ${ }^{2}$ Department of Chemistry, National College, Tiruchirappalli - 620 001, India \\ *E-mail address: drgtnarayanan@gmail.com
}

\begin{abstract}
A series of some aryl 1,3-oxazine-4-thione derivatives have been synthesized by 1-methyl imidazole catalyzed three component one pot synthetic method in room temperature. The purities of these thiones were studied by their physical constants and spectroscopic data. The infrared and ${ }^{13} \mathrm{C}$ NMR spectral data of $\mathrm{CN}$ and $\mathrm{CS}$ were correlated with Hammett substituent constants, $\mathrm{F}$ and $\mathrm{R}$ parameters using single and multi-linear regression analysis. From the results of statistical analysis, the effect of substituent on the spectral data was studied.
\end{abstract}

Keywords: 1,3-oxazine-4-thiones; IR spectra; ${ }^{13} \mathrm{C}$ NMR spectra; Hammett correlation

\section{INTRDUCTION}

Aryl 1,3-oxazine-4-thiones are pharmaceutical important heterocycles [1,2]. They possess various biological activities such as antimicrobial [3], hypolipidacmic [4], antidiabetic [5], anti-inflammatory [6], antimycobacterial [7], antithrombotic [8], antagonism to progesterone receptor [9], antitumor [10], antiviral [11], leucocyte clastase [12] and scrotonin reuptakes [13]. The ground state equilibration of organic compounds was established using spectroscopic data [14]. Infrared spectra was applied for prediction of $E s$ cis and s-trans conformers of styrenes[15], polyenes [16], chalcones [17], unsaturated aldehydes [18], acid chlorides [19], unsaturated esters [20], gauche and anti- form acyl halides and its esters [21]. The spatial arrangement of protons such as cis and trans of organic stereo chemical compounds [22] were studied using NMR spectra. Currently chemists and spectroscopic researchers [23-29] have paid much more interest for correlation of spectral data with Hammett substituent constants. Thirunarayanan and Ravi [30] have studied the effect of substituents of some pyrazoline-1-ethanones. Substituent effects on the spectral group frequencies of $9 H$-fluorenayl bromides were studied by Thirunarayanan [31]. Sakthinathan et al., have investigated the effect of substituents on naphthyl based pyrazoline derivatives [32]. Sasikala et al., [22] have evaluated the effect of substituents and antimicrobial activities of some 5-bromo-2-thienyl based pyrazolines. The spectral correlation of infrared and nuclear magnetic resonance spectra of $E$-imines have been predicted by Sakthinathan et. al. and Suresh et al., [33,34]. Thirunarayanan and Sekar have investigated the substituent effects on the IR and NMR spectral frequencies of some 3-(3,4- 
dichlorophenyl)-pyrazoline carbothiomides [35]. Spectral correlation study was first reported on the Tröger's bases by Thirunarayanan and Suresh [36]. Janaki et al, Vanangamudi et al, Subramanian et al. and Thirunarayanan et al., [37-40] have been investigated the effects of substituents on the spectral data of various chalcones. Thirunarayanan have studied the effect of substituents on the oxazine 2-amines by IR and NMR spectral data [27]. Recently, Thirunarayanan et al., [28] have studied the effect of substituents on aryl hydrazides by their infrared and nmr spectral data. Within the above view, there is no report available for the study of spectral correlation analysis on 1, 3-oxazine-4-thiones. Therefore the authors have taken efforts to synthesis and recorded the IR and NMR spectra for studying spectral correlation of above title compounds.

\section{EXPERIMENTAL}

\section{1. General}

All chemicals and solvents used in this present study were procured from SigmaAldrich and Merck companies. The infrared spectra of all oxazine imines have been recorded in SHIMADUZ Fourier Transform IR spectrophotometer using $\mathrm{KBr}$ disc. The NMR spectra of all compounds were recorded in BRUKER AV 400 type spectrometer, Using $\mathrm{CDCl}_{3}$ as a solvent, $100 \mathrm{MHz}$ frequency was applied for recording ${ }^{13} \mathrm{C}$ NMR spectra, taking TMS as standard.

\section{2. Synthesis of 1, 3-oxazine-4-thiones}

The title 1,3-oxazine-4-thiones were synthesized and their purities were checked by literature method [41]. The general structure of the aryl 1,3-oxazine-4-thions are shown in Fig. 1.

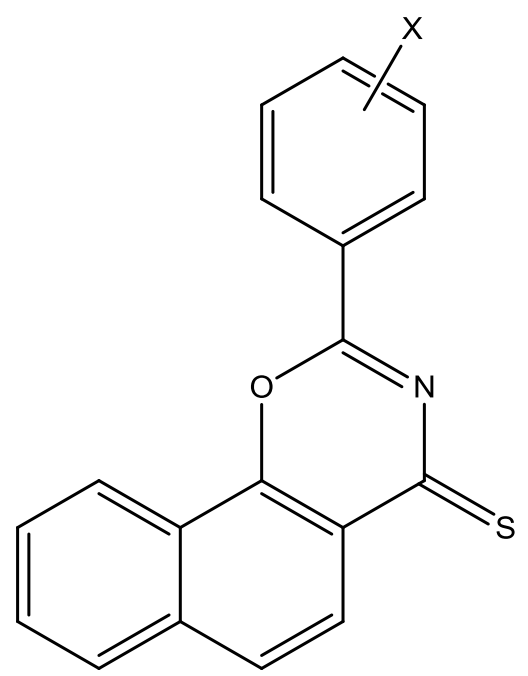

$\mathrm{X}=\mathrm{H}, 3-\mathrm{Br}, 4-\mathrm{Br}, 3-\mathrm{Cl}, 4-\mathrm{Cl}, 4-\mathrm{OCH}_{3}, 4-\mathrm{CH}_{3}, 3-\mathrm{NO}_{2}, 4-\mathrm{NO}_{2}$

Fig. 1. 2-(substituted phenyl)-4H-naphtho[2,1-e][1,3]oxazine-4-thiones. 


\section{RESULTS AND DISCUSSION}

In the present study, the authors have evaluated the effect of substituents on the infrared $\nu \mathrm{C}=\mathrm{N}, \mathrm{C}=\mathrm{S}\left(\mathrm{cm}^{-1}\right)$ stretches, ${ }^{13} \mathrm{C}$ NMR $\delta \mathrm{C}=\mathrm{N}$ and $\mathrm{C}=\mathrm{S}(\mathrm{ppm})$ chemical shifts of 2(substituted phenyl)-4H-naphtho[2,1-e][1,3]oxazine-4-thiones by Hammett correlation (Table $1)$.

Table 1. The infrared and ${ }^{13} \mathrm{C}$ NMR spectral data of 2-(substituted phenyl)- $4 H$-naphtho[2,1-e] $(1,3)$ oxazine-4-thiones.

\begin{tabular}{|c|c|c|c|c|c|}
\hline \multirow{2}{*}{ Sl. No. } & \multirow{2}{*}{$\mathbf{X}$} & \multicolumn{2}{|c|}{ IR (v, $\left.\mathbf{c m}^{-\mathbf{1}}\right)$} & \multicolumn{2}{c|}{${ }^{\mathbf{1 3}} \mathbf{C} \mathbf{N M R}(\boldsymbol{\delta}, \mathbf{p p m})$} \\
\cline { 3 - 6 } & & $\mathbf{C N}$ & $\mathbf{C S}$ & $\mathbf{C N}$ & $\mathbf{C S}$ \\
\hline $\mathbf{1}$ & $\mathrm{H}$ & 1655 & 1194 & 164.23 & 197.76 \\
\hline $\mathbf{2}$ & $3-\mathrm{Br}$ & 1658 & 1189 & 164.55 & 197.08 \\
\hline $\mathbf{3}$ & $4-\mathrm{Br}$ & 1666 & 1176 & 164.97 & 198.92 \\
\hline $\mathbf{4}$ & $3-\mathrm{Cl}$ & 1656 & 1178 & 164.76 & 197.16 \\
\hline $\mathbf{5}$ & $4-\mathrm{Cl}$ & 1662 & 1182 & 164.48 & 199.14 \\
\hline $\mathbf{6}$ & $4-\mathrm{OCH}_{3}$ & 1648 & 1169 & 164.26 & 197.02 \\
\hline $\mathbf{7}$ & $4-\mathrm{CH}_{3}$ & 1672 & 1174 & 165.49 & 198.09 \\
\hline $\mathbf{8}$ & $3-\mathrm{NO}_{2}$ & 1668 & 1198 & 165.96 & 198.06 \\
\hline $\mathbf{9}$ & $4-\mathrm{NO}_{2}$ & 1663 & 1206 & 165.54 & 197.97 \\
\hline
\end{tabular}

In infrared spectral correlation study, the Hammett equation was taken as,

$$
v=\rho \sigma+v_{0}
$$

where $v$ is the frequency for the substituted system, $\rho$ is the reaction constants in terms of intercept, $\sigma$ is the substituent constants and $v_{0}$ is the frequency for the parent member of the series.

The results of statistical analysis [14-17,21-28,31-46] were presented in Table 2. From the Table 2, the correlation of $\mathrm{vC}=\mathrm{N}, \mathrm{C}=\mathrm{S}\left(\mathrm{cm}^{-1}\right)$ stretches with Hammett substituent constants and $\mathrm{F}$ and $\mathrm{R}$ values are satisfactory along with positive $\rho$ values. Comparatively, the $\mathrm{C}=\mathrm{S}$ stretches were gave significant correlation than $v \mathrm{C}=\mathrm{N}$ stretches.

In ${ }^{13} \mathrm{C}$ nuclear magnetic resonance spectral correlations, the Hammett equation was used in the form as shown in (2):

$$
\log \delta=\log \delta_{0}+\rho \sigma
$$

where $\delta_{0}$ is the chemical shift of the corresponding parent compound. 
The results of statistical analysis of chemical shifts (ppm) of $\delta \mathrm{C}=\mathrm{N}, \mathrm{C}=\mathrm{S}[21-40,42-46]$ thiones were presented in Table 2. From the Table 2, the correlation of chemical shifts (ppm) of $\delta \mathrm{C}=\mathrm{N}$ with Hammett substituent constants and $\mathrm{F}$ and $\mathrm{R}$ values are satisfactory. The correlation of $\delta \mathrm{C}=\mathrm{S}$ with Hammett substituent constants and $\mathrm{F}$ and $\mathrm{R}$ values seems poor. This is due to the inability of effects of substituents on the chemical shifts through resonance or conjugation as shown in the Fig. 2. All correlations gave positive $\rho$ values. This inferred the normal substituent effects operates in all systems.

Some of the individual correlations were fail in the statistical analysis for the correlation of chemical shifts ( $\mathrm{ppm}$ ) of $\delta \mathrm{C}=\mathrm{N}, \mathrm{C}=\mathrm{S}$. while seeking this with multi-parameter correlation with Swain-Lupton's [42] parameters, they gave satisfactory correlations. The generated multi-regression analysis equations are shown in (3-10).

Table 2. Results of statistical analysis of IR and ${ }^{13} \mathrm{C}$ NMR spectral values of 2-(substituted phenyl)$4 H$-naphtho[2,1-e] $(1,3)$ oxazine-4-thione with Hammett $\sigma, \sigma^{+}, \sigma_{\mathrm{I}}, \sigma_{\mathrm{R}}$ constants,

$\mathrm{F}$ and $\mathrm{R}$ parameters.

\begin{tabular}{|c|c|c|c|c|c|c|c|}
\hline Frequency & Constant & $\mathbf{r}$ & $\mathbf{I}$ & $\rho$ & $\mathbf{S}$ & $\mathbf{n}$ & Correlated derivatives \\
\hline \multirow{6}{*}{$\begin{array}{c}v C N \\
\left(\mathrm{~cm}^{-1}\right)\end{array}$} & $\sigma$ & 0.903 & 1659.22 & 6.553 & 7.40 & \multirow{6}{*}{9} & \multirow{6}{*}{$\begin{array}{c}\mathrm{H}, 3-\mathrm{Br}, 4-\mathrm{Br}, 3-\mathrm{Cl} \\
4-\mathrm{Cl}, 4-\mathrm{OCH}_{3}, 4-\mathrm{CH}_{3} \\
\text { 2- } \mathrm{NO}_{2}, 4-\mathrm{NO}_{2}\end{array}$} \\
\hline & $\sigma^{+}$ & 0.904 & 1659.68 & 6.200 & 7.18 & & \\
\hline & $\sigma_{\mathrm{I}}$ & 0.902 & 1659.86 & 2.717 & 7.88 & & \\
\hline & $\sigma_{\mathrm{R}}$ & 0.905 & 1663.18 & 23.173 & 6.56 & & \\
\hline & $\mathrm{F}$ & 0.901 & 1659.41 & 3.980 & 7.85 & & \\
\hline & $\mathrm{R}$ & 0.905 & 1663.54 & 18.407 & 6.72 & & \\
\hline \multirow{6}{*}{$\begin{array}{c}v C S \\
\left(\mathrm{~cm}^{-1}\right)\end{array}$} & $\sigma$ & 0.978 & 1178.42 & 26.381 & 8.22 & \multirow{6}{*}{9} & \multirow{6}{*}{$\begin{array}{c}\mathrm{H}, 3-\mathrm{Br}, 4-\mathrm{Br}, 3-\mathrm{Cl}, \\
\text { 4-Cl, 4-OCH}, 4-\mathrm{CH}_{3} \\
\text { 2- } \mathrm{NO}_{2}, 4-\mathrm{NO}_{2}\end{array}$} \\
\hline & $\sigma^{+}$ & 0.973 & 1181.58 & 18.116 & 8.93 & & \\
\hline & $\sigma_{\mathrm{I}}$ & 0.904 & 1177.02 & 21.461 & 11.88 & & \\
\hline & $\sigma_{R}$ & 0.988 & 1191.18 & 61.419 & 6.09 & & \\
\hline & $\mathrm{F}$ & 0.904 & 1176.37 & 23.528 & 11.88 & & \\
\hline & $\mathrm{R}$ & 0.988 & 1192.51 & 51.251 & 6.26 & & \\
\hline \multirow{6}{*}{$\begin{array}{c}\delta \mathrm{CN} \\
(\mathbf{p p m})\end{array}$} & $\sigma$ & 0.958 & 164.66 & 0.981 & 0.53 & \multirow{6}{*}{9} & \multirow{6}{*}{$\begin{array}{c}\mathrm{H}, 3-\mathrm{Br}, 4-\mathrm{Br}, 3-\mathrm{Cl}, \\
4-\mathrm{Cl}, 4-\mathrm{OCH}_{3}, 4-\mathrm{CH}_{3} \\
\text { 2- } \mathrm{NO}_{2}, 4-\mathrm{NO}_{2}\end{array}$} \\
\hline & $\sigma^{+}$ & 0.954 & 164.78 & 0.669 & 0.55 & & \\
\hline & $\sigma_{\mathrm{I}}$ & 0.903 & 164.56 & 0.919 & 0.61 & & \\
\hline & $\sigma_{R}$ & 0.969 & 165.15 & 2.414 & 0.47 & & \\
\hline & $\mathrm{F}$ & 0.904 & 164.49 & 1.144 & 0.59 & & \\
\hline & $\mathrm{R}$ & 0.962 & 165.18 & 1.832 & 0.51 & & \\
\hline \multirow{6}{*}{$\begin{array}{c}\delta C S \\
(\mathbf{p p m})\end{array}$} & $\sigma$ & 0.812 & 197.84 & 0.241 & 0.80 & \multirow{6}{*}{9} & \multirow{6}{*}{$\begin{array}{c}\mathrm{H}, 3-\mathrm{Br}, 4-\mathrm{Br}, 3-\mathrm{Cl} \\
\text { 4-Cl, 4- } \mathrm{OCH}_{3}, 4-\mathrm{CH}_{3} \\
\text { 2- } \mathrm{NO}_{2}, 4-\mathrm{NO}_{2}\end{array}$} \\
\hline & $\sigma^{+}$ & 0.882 & 197.84 & 0.347 & 0.81 & & \\
\hline & $\sigma_{I}$ & 0.810 & 197.76 & 0.375 & 0.80 & & \\
\hline & $\sigma_{R}$ & 0.825 & 198.01 & 1.069 & 0.78 & & \\
\hline & $\mathrm{F}$ & 0.811 & 197.77 & 0.355 & 0.81 & & \\
\hline & $\mathrm{R}$ & 0.828 & 198.05 & 1.008 & 0.78 & & \\
\hline
\end{tabular}

$\mathrm{r}=$ correlation coefficient $\mathrm{I}=$ intercept $; \rho=$ slope $; \mathrm{s}=$ standard deviation; $\mathrm{n}=$ number of correlated derivatives 


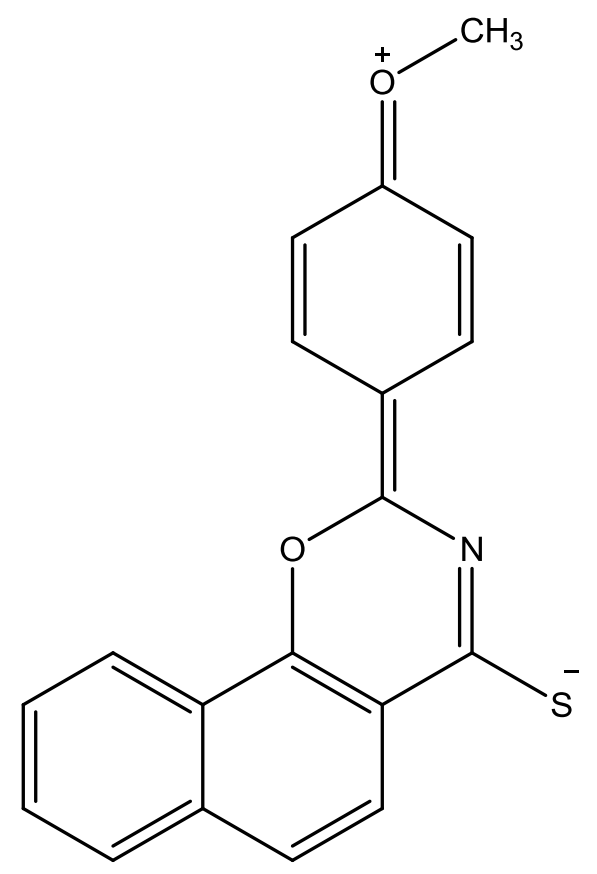

Fig. 2. The resonance-conjugative structure.

$$
\begin{aligned}
& v \mathrm{CN}\left(\mathrm{cm}^{-1}\right)=1664.36( \pm 5.169)+2.810( \pm 1.043) \sigma_{\mathrm{I}}+24.455( \pm 10.7176) \sigma_{\mathrm{R}} \\
& (\mathrm{r}=0.956, \mathrm{n}=9, \mathrm{P}>95 \%) \\
& v \mathrm{CN}\left(\mathrm{cm}^{-1}\right)=1664.18( \pm 5.688)+1.509( \pm 0.114) \mathrm{F}+18.949( \pm 7.120) \mathrm{R} \\
& (\mathrm{r}=0.952, \mathrm{n}=9, \mathrm{P}>95 \%) \\
& v \operatorname{CS}\left(\mathrm{cm}^{-1}\right)=1187.62( \pm 4.516)+8.447( \pm 0.911) \sigma_{\mathrm{I}}+57.623( \pm 12.853) \sigma_{\mathrm{R}} \\
& (\mathrm{r}=0.990, \mathrm{n}=9, \mathrm{P}>95 \%) \\
& v \mathrm{CS}\left(\mathrm{cm}^{-1}\right)=1188.41( \pm 4.927)+9.687( \pm 0.991) \mathrm{F}+47.777( \pm 11.046) \mathrm{R} \\
& (\mathrm{r}=0.989, \mathrm{n}=9, \mathrm{P}>95 \%) \\
& \delta \mathrm{CN}(\mathrm{ppm})=164.97( \pm 0.366)+0.417( \pm 0.073) \sigma_{\mathrm{I}}+2.223( \pm 1.043) \sigma_{\mathrm{R}} \\
& (\mathrm{r}=0.949, \mathrm{n}=9, \mathrm{P}>90 \%) \\
& \delta \mathrm{CN}(\mathrm{ppm})=164.89( \pm 0.427)+6.684( \pm 0.083) \mathrm{F}+1.586( \pm 0.062) \mathrm{R} \\
& (\mathrm{r}=0.967, \mathrm{n}=9, \mathrm{P}>95 \%) \\
& \delta \mathrm{CS}(\mathrm{ppm})=197.95( \pm 0642)+0.149( \pm 0.012) \sigma_{\mathrm{I}}+1.002( \pm 0.177) \sigma_{\mathrm{R}} \\
& (\mathrm{r}=0.925, \mathrm{n}=9, \mathrm{P}>90 \%) \\
& (\mathrm{r}=0.928, \mathrm{n}=9, \mathrm{P}>90 \%)
\end{aligned}
$$




\section{CONCLUSIONS}

There are nine aryl 1,3-oxazine-4-thione derivatives have been synthesized by 1-methyl imidazole catalyzed three component one pot synthetic method in room temperature. The purities of these thiones were studied by their physical constants and spectroscopic data. The infrared and ${ }^{13} \mathrm{C}$ NMR spectral data of $\mathrm{CN}$ and CS were correlated with Hammett substituent constants, $\mathrm{F}$ and $\mathrm{R}$ parameters using single and multi-linear regression analysis. The infrared $\nu \mathrm{CN}$ and $\nu \mathrm{CS}$ stretches $\left(\mathrm{cm}^{-1}\right)$ were correlated satisfactorily with Hammett substituent constants, $\mathrm{F}$ and $\mathrm{R}$ parameters. Satisfactory correlation was obtained for the ${ }^{13} \mathrm{C}$ chemical shifts of $\delta \mathrm{CN}(\mathrm{ppm})$ of thiones with Hammett substituent constants, $\mathrm{F}$ and $\mathrm{R}$ parameters. The $\delta \mathrm{CS}(\mathrm{ppm})$ of thiones were failed in correlation. In multiple correlation all spectral frequencies gave satisfactorily correlation with Swain-Lupton's constants.

\section{References}

[1] D. A. Brown, C. J. Powers, Bioorg. Med. Chem. 3 (1995) 1091-1097.

[2] M. Gutschow, L. Kuerschner, U. Neumann, M. Pietsch, R. Loser, N. Koglin, K. Eger, J. Med. Chem. 42 (1999) 5437-5447.

[3] A. N. Mayekar, H. S. Yathirajan, B. Narayana, B. K. Sarojini, N. Suchetha Kumari and W. T. A. Harrison, Int. J. Chem. 3 (1) (2011) 74-86.

[4] G. R. Madhavan, R. Chakrabarti, K. A. Reddy, B. M. Rajesh, V. Balaju, P. B. Rao, R. Rajagopalan, J. Iqbal, Bioorg. Med. Chem. 14 (2006) 584-591

[5] K. Balbir, K. Ramandeep, Arkivoc. 15 ( 2007) 315-323

[6] P. W. Hsieh, R. F. Chang, H. C. Chang, W. P. Cheng, C. L. Chiang, L. F. Zeng, K. H. Lin, C. Y. Wu, Bioorg. Med. Chem. Lett. 14 (2004) 4751-4754

[7] K. Waisser, J. Gregor, L. Kubikova, V. Klimesova, J. Kunes, M. Machacek, J. Kaustova, Eur. J. Med. Chem. 35 (2000) 733-741

[8] P. W. Hsieh, T. L. Hwang, C. C. Wu, F. R. Chang, T. W. Wang, Y. C. Wu, Bioorg. Med. Chem. Lett. 15 (2005) 2786-2789

[9] J. C. Kern, E. A. Terefenko, A. Fensome, R. Unwalla, J. Wrobel, Y. Zhu, J. Cohen, R. Winneker, Z. Zhang, P. Zhang, Bioorg. Med. Chem. Lett. 17 (2007) 189-192

[10] A. Bolognese, G. Correale, M. Manfra, A. Lavecchia, O. Mazzoni, E. Novellino, V. Barone, P. La Colla, R. Loddo, J. Med. Chem. 45 (2002) 5217-5223

[11] R. L. Jarvest, S. C. Connor, J. G. Gorniak, L. J. Jennings, H. T. Serafinowska and A. West., Bioorg. Med. Chem. Lett. 7 (1997) 1733-1738

[12] E. Colson, J. Wallach and M. Hauteville, Biochimie 87(2005)223-230

[13] P. J. Atkinson, et al., Bioorg. Med. Chem. Lett. 15 (2005) 737-741

[14] G. Thirunarayanan, M. Gopalakrishnan, G. Vanangamudi, Spectrochim. Acta 67A (2007) 1106-1612.

[15] A. Perjessy, M. Laucova, Coll. Czech. Chem. Commun. 36 (1971) 2944-22950. 
[16] W. F. Winecoff, D. W. Boykin Jr., J. Org. Chem. 37 (4) (1972) 676.

[17] G. Thirunarayanan, P. Ananthakrishna Nadar, Asian J. Chem. 14(3-4) (2002) 1518-1522.

[18] D. Seebach, R. Gilmour, U. Grošelj, G. Deniau, C. Sparr, M. O. Ebert, A. K. Beck, Helvi. Chem. Acta. 93 (2010) 603-634.

[19] T. A. Foglia, P. A. Barr and M. J. Idacavage, J. Org. Chem. 41 (1976) 3452-3455.

[20] V. Všetečka, J. Pecka, M. Procházka, Collect. Czech. Chem. Commun. 47 (1982) 277-285.

[21] G. Thirunarayanan, G. Vanangamudi, V. Sathiyendiran, K. Ravi, Indian J. Chem. 50B(4) (2011) 593-604.

[22] G. Thirunarayanan, P. Mayavel, K. Thirumurthy, S. Dineshkumar, R. Sasikala, P. Nisha, A. Nithyaranjani, European Chem. Bull. 2(9) (2013) 598-605.

[23] K. Rajasekharan, C. Gnansekaran, J. Chem. Soc. Perkin Trans II (1987) 263-266.

[24] K. Rajasekharan, C. Gnansekaran, Indian J. Chem. 25A (1986) 64-66.

[25] V. Mala, K. Sathiyamoorthy, S. P. Sakthinathan, D. Kamalakkannan, R. Suresh, G. Vanangamudi, G. Thirunarayanan, Q-Science Connect, 2013:7;

DOI http://dx.doi.org/10.5339,2013.7

[26] G. Vanangamudi, M. Subramanian, P. Jayanthi, R. Arulkumaran, D. Kamalakkannan G. Thirunarayanan, Arabian J. of Chemistry, (2011). doi:10.1016/j.arabjc.2011.07.019

[27] G. Thirunarayanan, International Letters of Chemistry, Physics and Astronomy 4 (2014) 109-116.

[28] G. Thirunarayanan, K.G. Sekar, R. Lakshmi Narayanan, International Letters of Chemistry, Physics and Astronomy 13(1) (2014) 88-94.

[29] M. A. Khalilzadeh, I. Yavari, Z. Hossaini and H. Sadeghifar, Monastch Chem. 140 (2009) 467-471.

[30] R. Arulkumaran, S. Vijayakumar, R. Sundararajan, S. P. Sakthinathan, D. Kamalakkannan, R. Suresh, K. Ranganathan, G. Vanangamudi, G. Thirunarayanan, International Letters of Chemistry, Physics and Astronomy 4 (2012) 17-38.

[31] G. Thirunarayanan, K. Ravi, International Letters of Chemistry, Physics and Astronomy 14 (2013) 44-57.

[32] G. Thirunarayanan, International Letters of Chemistry, Physics and Astronomy 9(2) (2013) 152-161.

[33] S. P. Sakthinathan, G. Vanangamudi, G. Thirunarayanan, Spectrochim. Acta 95A (2012) 693-700.

[34] S. P. Sakthinathan, R. Suresh, V. Mala, K. Sathiyamoorthi, D. Kamalakkannan, K. Ranganathan, S. John Joseph, G. Vanangamudi, G. Thirunarayanan, Int. J. Sci. Res. Know. 1(11) (2013) 472-483. 
[35] R. Suresh, D. Kamalakkannan, K. Ranganathan, R. Arulkumaran, R. Sundararajan, S.P. Sakthinathan, S. Vijayakumar, K. Sathiyamoorthi, V. Mala, G. Vanangamudi, K. Thirumurthy, P. Mayavel, G. Thirunarayanan, Spectrochim. Acta. 101A (2013) 239-248.

[36] G. Thirunarayanan, K. Sekar, Organic Chem: An Indian J. 9(12) (2013) 483-492.

[37] G. Thirunarayanan, M. Suresh, International Letters of Chemistry, Physics and Astronomy 1 (2014) 67-73.

[38] P. Janaki, K. G. Sekar, G. Thirunarayanan, Journal of Saudi Chem. Soc. (2012) Doi.10.1016/j.jscs.2012.11.013

[39] G. Vanangamudi, M. Subramanian, G. Thirunarayanan, Arabian J. Chem. (2013)

DOI: 10.1016/j.arabjc.2013.03.006,

[40] M. Subramanian, G. Vanangamudi, G. Thirunarayanan, Spectrochim. Acta 110A (2013) 116-123.

[41] G. Thirunarayanan, G. Vanangamudi, M. Subramanian, Organic Chemistry: An Indian $J$ 9(1) (2012)1-16.

[42] Swain C. G., Lupton E. C. Jr., J. Am. Chem. Soc. 90 (1968) 4328-4337.

[43] G. Thirunarayanan, K. G. Sekar, International Letters of Chemistry, Physics and Astronomy 10(1) (2013) 18-34.

[44] K. G. Sekar, G. Thirunarayanan, International Letters of Chemistry, Physics and Astronomy 8(3) (2013) 249-258

[45] S. Vijayakumar, R. Arulkumaran, R. Sundararajan, S. P. Sakthinathan, R. Suresh, D. Kamalakkannan, K. Ranganathan, K. Sathiyamoorthi, V. Mala, G. Vanangamudi, G. Thirunarayanan, International Letters of Chemistry, Physics and Astronomy 9(1) (2013) 68-86.

[46] S. P. Sakthinathan, R. Suresh, V. Mala, K. Sathiyamoorthi, D. Kamalakkannan, K. Ranganathan, R. Arulkumaran, S. Vijayakumar, R. Sundararajan, G. Vanangamudi, G. Thirunarayanan, International Letters of Chemistry, Physics and Astronomy 6 (2013) 77-90. 